\title{
Ergodic Properties of the Lozi Mappings
}

\author{
P. Collet and Y. Levy \\ Centre de Physique Théorique, Ecole Polytechnique, F-91128 Palaiseau Cédex, France
}

\begin{abstract}
In this paper, we construct the Bowen-Ruelle measure for the Lozi mapping, an almost everywhere hyperbolic diffeomorphism of the plane. We also derive some of its properties which are similar to those of an axiom $A$ system.
\end{abstract}

\section{Introduction}

The Lozi mapping $T$ is a homeomorphism of $\mathbb{R}^{2}$ given by

$$
\left(\begin{array}{l}
x \\
y
\end{array}\right) \rightarrow\left(\begin{array}{c}
1+y-a|x| \\
b x
\end{array}\right) .
$$

For some values of $a$ and $b$, Lozi [Lo] observed complicated behaviour for the trajectories of this system. For $b=0.5$ and $a=1.7$ one observes numerically a strange attractor, which is very similar to the attractor of the Henon map [He]. The main advantage of the Lozi map over the Henon map is that one can prove hyperbolicity without much effort. This is the main reason why so little is known for the Henon map, where hyperbolicity is believed to occur only on Cantor-like sets of parameters. Our opinion is that the Lozi mapping is an intermediate stage between the Axiom $A$ dynamical systems and more complicated systems like the Henon map. As we shall see below, its dynamical structure is more complicated than in the Axiom $A$ systems, although some detailed ergodic properties are the same. The Lozi map is rather similar to Sinai's billiards, and in this article, we shall use this analogy. In particular, the discontinuity of the differential allows the uniform hyperbolicity as in the billiards case. A proof of hyperbolicity for the Lozi map was first given by Misiurewicz [M]. He also derived many important consequences which will be described below.

This article is devoted to the investigation of the metric properties of the Lozi map. In the next paragraph, we briefly describe some properties of the map which will be needed later on. Most of them were known before. In the third paragraph we construct an invariant measure; its ergodic properties (absolute continuity with 
respect to the Lebesgue measure in the unstable direction, ergodicity, K-property, Bowen-Ruelle property, Bernoulli character) are derived in Sect. IV.

Similar results were obtained by Rychlik [Ry] using a different proof. We learned that Young [Y1] has proven similar results for piecewise $C^{2}$ hyperbolic maps.

\section{Notations - General Properties}

We set some notations which will be of constant use in this paper. $\mathscr{M}$ is the Borel $\sigma$-algebra of $\mathbb{R}$. If $A$ is a measurable subset of $\mathbb{R}^{2}, \mathscr{M}_{A}$ is the corresponding factor sub $\sigma$-algebra. $l$ and $m$ are the 1-dim and 2-dim Lebesgue measure. $d$ is the Euclidean distance in $\mathbb{R}^{2}$. We shall sometimes use the notations \pm and $\mp$. We adopt the values of $[\mathrm{M}]$ for $a$ and small values for $b$. For a partition $\xi$ of the space $X, \xi(x), x \in X$ is the atom of $\xi$ containing $x$. Let $S_{0}^{-}=O y, S_{0}^{+}=O x=T S_{0}^{-}$; $S_{n}^{ \pm}=T^{ \pm n} S_{0}^{ \pm}, n \in \mathbb{N}$, are finite broken lines. Note that $T^{ \pm n}$ is singular on $S_{n}^{\mp}, n \in \mathbb{N}$.

The fields of stable (unstable) directions $E^{s}(\cdot)\left[E^{u}(\cdot)\right]$ are defined outside $\bigcup_{n \geqq 0} S_{n}^{-}\left(\bigcup_{n \geqq 0} S_{n}^{+}\right)$(cf. [M]). If $\theta^{s}(x)$ and $\theta^{u}(x)$ are their angles with respect to the $x$ axis, we have continuous fraction expansions for $\tan \theta^{s}(x)$ and $\tan \theta^{u}(x)$ which are given by

$$
\tan \theta^{u}(x)=\frac{-b}{a \cdot \varepsilon\left(T^{-1} x\right)-\tan \theta^{u}\left(T^{-1} x\right)}, \quad \tan \theta^{s}(x)=a \cdot \varepsilon(x)+\frac{b}{\tan \theta^{s}(T x)},
$$

where $\varepsilon(x)$ is the sign of $x$. These formulae express the fact that $E^{s}(\cdot)$ and $E^{u}(\cdot)$ are invariant fields. If $b / a$ is small enough, the above continuous fraction expansions are convergent. We shall denote by $\lambda(x)$ the expansion factor in the direction $E^{u}(x)$, i.e. this is the length of the image by $D T_{x}$ of the unit vector in the direction $E^{u}(x)$. It is easy to verify that

$$
\lambda(x)=\frac{\left[a^{2}+b^{2}+\tan ^{2}\left(\theta^{u}(x)\right)-2 a \varepsilon(x) \tan \theta^{u}(x)\right]^{1 / 2}}{\left(1+\tan ^{2} \theta^{u}(x)\right)^{1 / 2}} .
$$

Let $W^{s}(x)$ [respectively $W^{u}(x)$ ] be the global stable (respectively unstable) manifold of $x$. The maximal smooth component $W_{\mathrm{loc}}^{s}(x)\left[W_{\mathrm{loc}}^{u}(x)\right]$ of $W^{s}(x)$ $\left[W^{u}(x)\right]$ containing $x$ will be called the local stable (local unstable) manifold of $x . x$ splits $W_{\text {loc }}^{u}(x)$ into two "semi local unstable manifolds."

Let $X$ be the fixed point of $T$ with positive coordinates. $Z$ is the intersection of the positive $x$ axis with $\overline{W_{\text {loc }}^{u}(X)}$. We shall denote by $F$ the triangle defined by the points $Z, T(Z), T^{2}(Z)$ (see $[\mathrm{M}]$ ). $\Omega$ will denote the strange attractor of $T$ which is equal to $\bigcap_{0}^{\infty} T^{n}(F)[\mathrm{M}]$. We shall denote by $\lambda$ and $\lambda_{+}$the infimum and the supremum of $\lambda(x)$ for $x$ in the set $F \backslash \bigcup_{-\infty}^{+\infty} S_{l}^{ \pm}: \lambda>0$. lemma

It is now easy, using the continuous fraction expansion, to verify the following

Lemma II.1. Let $x$ and $y$ in $F$ be such that for $0 \leqq j \leqq q, T^{j}(x)$ and $T^{j}(y)$ are on the same side of $S_{0}$. Then 
i) The angle between $W_{\mathrm{loc}}^{s}(x)$ and $W_{\mathrm{loc}}^{s}(y)$ (if they are defined) is bounded by $2\left(\frac{b}{\lambda}\right)^{q-1}$
ii) $\left|\frac{\lambda(x)}{\lambda(y)}-1\right| \leqq 2\left(\frac{b}{\lambda}\right)^{q-1}$.

We also observe that the angle between two local stable manifolds is between $\frac{2 \pi}{5}$ and $-\frac{2 \pi}{5}$ if $a<2$, and the angle between a local stable and a local unstable manifold is greater than $\frac{\pi}{5}$.

We now introduce a set $H$ which is more convenient for our purposes than the set $F$. This set was already used by Misiurewicz but we recall here its properties.

Lemma II.2. For $b$ small enough, there is a polygon $H$ such that

i) $\mathrm{THCH}$,

ii) $\Omega \subset H$,

iii) The boundary of $H$ is contained in $T^{q} W_{\mathrm{loc}}^{u}(X) \cup W_{\mathrm{loc}}^{s}(X)$ for some $q>0$.

iv) $T^{r} F \subset H$ for some positive $r$.

Proof. We adopt the notations of [M]. $H_{0}$ is the triangle $X Z P$, where $P=W_{\mathrm{loc}}^{s}(X)$ $\cap(Z, T(Z))$ and $H=\bigcup_{n=0}^{\infty} T^{n}\left(H_{0}\right)$. ( $T$ is denoted $f$ in $[\mathrm{M}]$.)

In Proposition 2 of $[\mathrm{M}]$, it is shown that there is a positive integer $p$ such that

$$
H=\bigcup_{n=0}^{p} T^{n}\left(H_{0}\right)
$$

Moreover, in the same Proposition 2, it is established that

$$
T^{p} F \subset H \text { and } T H \subset H \text {. Q.E.D. }
$$

We shall use the following mixing property derived by $[\mathrm{M}]$.

Proposition II.3. $(\Omega, T)$ is topologically mixing, i.e.: if $A, B \subset \mathbb{R}^{2}$ are open, then

$$
(A \cap \Omega \neq \emptyset, B \cap \Omega \neq \emptyset) \Rightarrow\left(\exists N, n>N \Rightarrow T^{n} A \cap B \cap \Omega \neq \emptyset\right) .
$$

We investigate now the absolute continuity of the unstable foliation. Let $W^{1}$ and $W^{2}$ be two local unstable manifolds in $\Omega$. We define a map $P=P_{W^{1} W^{2}}$ from $W^{1}$ to $W^{2}$ by

$$
x \in W^{1} \rightarrow P(x)=W_{\mathrm{loc}}^{s}(x) \cap W^{2} \quad \text { if } \quad W_{\mathrm{loc}}^{s}(x) \cap W^{2} \neq \emptyset .
$$

$P$ is defined on $\mathscr{D}(P)=\left\{x \in W^{1} \mid W_{\text {loc }}^{s}(x) \cap W^{2} \neq \emptyset\right\}$.

Proposition II.4. Given $W^{1}$ and $W^{2}$ as above there is a constant $L_{1}>0$ which is $\mathcal{O}(1)$ such that for any Borel subset $A$ of $W^{1}, A \subset \mathscr{D}(P)$,

$$
\left(1-L_{1}\left(d\left(W^{1}, W^{2}\right)\right)^{1 / 3}\right) l(A) \leqq l(P(A)) \leqq\left(1+L_{1}\left(d\left(W^{1}, W^{2}\right)\right)^{1 / 3}\right) l(A) .
$$

The proof is given in the appendix. 


\section{Construction of Invariant Measures}

Let $L_{0}=W_{\text {loc }}^{u}(X)$. We know from Misiurewicz's work [M] that $\Omega$ is the closure of $\bigcup_{n \geq 0} T^{n} L_{0}$. Therefore, it is natural to try to obtain an invariant measure by iterating the Lebesgue measure supported by $L_{0}$. We define a sequence $\left(\mu_{n}^{\prime}\right)_{n \in \mathbb{Z}}$ of probability measures on $\mathscr{M}_{\Omega}\left(\mu_{n}^{\prime}\right.$ has support in $\left.T^{n} L_{0}\right)$ by $\mu_{n}^{\prime}(A)=l\left(T^{-n} A \cap L_{0}\right) / l\left(L_{0}\right)$ for $A \in \mathscr{M}_{\Omega}$. The sequence $\left(\mu_{n}\right)_{n \in \mathbb{N}}$ defined by

$$
\mu_{n}=\frac{1}{n} \sum_{j=0}^{n-1} \mu_{j}^{\prime}
$$

is a sequence of probability measures on $\mathscr{M}_{\Omega}$. As $\Omega$ is compact one can extract a subsequence which vaguely converges to an invariant probability measure $\mu$ (cf. [B]). Let $A_{\varepsilon}^{ \pm}$denote the stripe of width $\varepsilon$ around $S_{0}^{ \pm}$.

In order to obtain some properties of the measure $\mu$, we shall first estimate the $\mu_{n}$-measures of $A_{\varepsilon}^{ \pm}$.

Proposition III.1. For any positive number $\tau<\left(1-\frac{1}{K \log \lambda}\right)$ ( $K$ is the integer appearing in Lemma III.2), there is a positive real number $\varepsilon_{0}$, such that for $0<\varepsilon \leqq \varepsilon_{0}$, we have $\mu_{n}\left(A_{\varepsilon}^{ \pm}\right) \leqq \varepsilon^{\tau}, \forall n \in \mathbb{N}$.

We give the proof for $A_{\varepsilon}=A_{\varepsilon}^{-}$. We shall first give some geometrical considerations. For $n \in \mathbb{N}, T^{n} L_{0}$ is a segment or a broken line. Let $J_{n}$ be the set of maximal smooth components contained in $T^{n} L_{0}$. For $M \in J_{n}$, the endpoints of $M$ belong to $S_{p} \cup S_{q}$ for some $p, q 1 \leqq p, q \leqq n+2$. We define $k(M)$ by $k(M)=\inf (p, q)$. For $M$ belonging to $J_{n}$, we shall denote by $R_{p}(M)$ the element of $J_{n-p}$ containing $T^{-p} M$ for $p \in \mathbb{Z}$. We now define recursively a finite sequence of integers $k_{i}(M)$ by

$$
\begin{gathered}
k_{0}(M)=0, \\
k_{1}(M)=k(M), \\
k_{i+1}(M)=k_{i}(M)+k\left(R_{k_{i}(M)}(M)\right) \quad \text { as long as } R_{k_{i}(M)}(M) \neq L_{0} .
\end{gathered}
$$

We shall write $k_{i}$ instead of $k_{i}(M)$ when there is no ambiguity. We now prove a lower bound on $k(\cdot)$.

Lemma III.2. Assume $b$ is small enough, there is an integer $K>4$ and $\theta>0$ such that if $M \in J_{n}, l(M)<\theta$, and $M \cap S_{0}^{-} \neq \emptyset$, then $k(M)>K$.

Proof. For $b$ small enough, there is an integer $K>2$ such that $0<p \leqq K$ implies $\left(S_{0}^{-} \cap F\right) \cap S_{p}^{-}=\emptyset$.

Let $\theta=\inf _{0<p \leqq K} d\left(S_{0}^{-} \cap F, S_{p}^{-} \cap F\right)$, then if $l(M)<\theta$, we have $k(M)>K$. Q.E.D.

We now come to the basic estimate. It is enough to prove the assertion for $\mu_{n}^{\prime}$, $n \in \mathbb{Z}$. The proof is recursive. Let $\tau$ be a number such that $0<\tau<1-\frac{1}{K \log \lambda}$. The estimate is obvious for $\mu_{-1}^{\prime}$, since $l\left(L_{0}\right)>1$; for $\mu_{n}^{\prime}, n \leqq-2$ and $\varepsilon$ small enough, $\mu_{n}^{\prime}\left(A_{\varepsilon}\right)=0$. From now on, we assume the bound has been already proven for $\mu_{p}^{\prime}$, $-1 \leqq p<n$. 
Let $\varrho, 0<\varrho<1$ be such that

$$
(1-\varrho)\left(1-\frac{1}{K \log \lambda}\right)>\tau \text {. }
$$

Note that such a $\varrho$ exists since $\tau<1-\frac{1}{K \log \lambda}$.

Let $E_{1}=\left\{M \in J_{n} \mid l(M)>4 \varepsilon^{o}\right\}$. If $M \in E_{1}, M$ is a straight segment of $T^{n} L_{0}$, therefore

$$
\frac{l\left(T^{-n}\left(A_{\varepsilon} \cap M\right)\right)}{l\left(T^{-n} M\right)}=\frac{l\left(A_{\varepsilon} \cap M\right)}{l(M)} \leqq \frac{4 \varepsilon}{4 \varepsilon^{\varrho}}=\varepsilon^{1-\varrho},
$$

since $l\left(A_{\varepsilon} \cap M\right) \leqq 4 \varepsilon$ (we have used the fact that the contraction coefficient by $T^{-n}$ is constant along $M$ ). Therefore,

$$
\sum_{M \in E_{1}} l\left(T^{-n}\left(A_{\varepsilon} \cap M\right)\right) \leqq \varepsilon^{1-\varrho} \sum_{M \in E_{1}} l\left(T^{-n} M\right) \leqq \varepsilon^{1-\varrho} l\left(L_{0}\right) .
$$

We now define a subset $E_{2}$ of $J_{n}$ by

$$
E_{2}=\left\{M \in J_{n}\left|\exists i \in \mathbb{N}, k_{i}(M)<c\right| \log \varepsilon \mid, l\left(R_{k_{i}}(M)\right)>4 \varepsilon^{o} \lambda^{-k_{i}(M)}\right\},
$$

where $c$ is a fixed number satisfying

$$
K(1-\varrho) / \log 2>c>(1-\varrho) / \log \lambda \quad\left(\text { note that } \frac{K \log \lambda}{\log 2}>1\right) .
$$

For $M \in E_{2}$ let $\sigma(M)$ be the smallest integer $i$ such that

$$
l\left(R_{k_{i}}(M)\right)>4 \varepsilon^{e} \hat{\lambda}^{-k_{i}(M)} .
$$

Let $p$ be a positive integer, and let $\tilde{M}$ be an element of $J_{n-p}$ such that $\tilde{M} \cap S_{0}^{-} \neq \emptyset$. We shall denote by $N(\tilde{M}, p)$ the number of elements $M$ of $J_{n}$ such that $T^{-p} M$ is included in $\tilde{M}$, and $k_{\sigma(M)} \geqq p$.

Let

$$
L_{p}=\sup _{0 \leqq k \leqq p} \sup _{\substack{\tilde{M} \in J_{n-k}-k \\ \tilde{M} \cap S_{0} \neq \emptyset}} N(\tilde{M}, k)
$$

Note that $L_{p}$ is a non-decreasing sequence. We shall now give an upper bound for $L_{p}$. For $\tilde{M}$ as above let $\tilde{M}_{L}$ (respectively $\tilde{M}_{R}$ ) be the segment $\tilde{M} \cap\{(x, y) \mid x \leqq 0\}$ [respectively $\tilde{M} \cap\{(x, y) \mid x \geqq 0\}]$. Assume moreover that the subset $E_{L}(p)$ of $E_{2}$ defined by $E_{L}(p)=\left\{M \in E_{2} \mid T^{-p} M \subset \tilde{M}_{L}, k_{\sigma(M)} \geqq p\right\}$ is non-empty. [If $E_{L}(p)$ is empty, then $E_{R}(p)$ is not empty or $L_{p}=0$.] Let $q$ be the smallest positive integer such that $T^{q} \tilde{M}_{L} \cap S_{0}^{-} \neq \emptyset$. There are two cases.

Case 1. $q<p$. Then $T^{q} \tilde{M}_{L} \in J_{n-p+q}$, and for any $M \in E_{L}(p)$, we have $R_{k_{i}}(M)=T^{q} \tilde{M}_{L}$ for some integer $i$. Moreover, $k_{i}(M)=p-q<p \leqq k_{\sigma(M)}$. This implies $i<\sigma(M)$, and

$$
l\left(T^{q} \tilde{M}_{L}\right) \leqq 4 \varepsilon^{e} \lambda^{-k_{i}(M)} .
$$


If $\varepsilon_{0}$ is small enough, we have $4 \varepsilon^{\varrho}<\theta$, and we can apply Lemma III.2 to conclude that $q>K$. Therefore,

$$
\begin{aligned}
\operatorname{card}\left(E_{L}(p)\right) & =\operatorname{card}\left\{M \in E_{2} \mid T^{-p+q} M \subset T^{q} \tilde{M}_{L}, k_{\sigma(M)} \geqq p\right\} \\
& \leqq \operatorname{card}\left\{M \in E_{L}(p) \mid T^{-p+q} M \subset T^{q} \tilde{M}_{L}, k_{\sigma(M)} \geqq p-K\right\} \\
& \leqq \sup _{0 \leqq k \leqq p-K} \sup _{\substack{\tilde{M}^{\prime} \in J_{n-k} \\
\tilde{M}^{\prime} \cap S_{0} \neq \emptyset}} N\left(\tilde{M}^{\prime}, k\right)=L_{p-K} .
\end{aligned}
$$

Case 2. $q \geqq p$. In this case we have $T^{p} \tilde{M}_{L} \in J_{n}$, and $\operatorname{card} E_{L}(p)=1$. Similarly, we define $E_{R}(p)$ by

$$
E_{R}(p)=\left\{M \in E_{2} \mid T^{-p} M \subset \tilde{M}_{R}, k_{\sigma(M)} \geqq p\right\},
$$

and we obtain as before $\operatorname{card} E_{R}(p)=1$ or $\operatorname{card} E_{R}(p) \leqq L_{p-K}$. Therefore, $N(\tilde{M}, p)$ $=\operatorname{card} E_{L}(p)+\operatorname{card} E_{R}(p) \leqq \sup \left(2,2 L_{p-K}\right)$, and we obtain the bound

$$
L_{p} \leqq 2^{1+p / K} .
$$

Let now $p$ be an integer such that $0 \leqq p \leqq c|\log \varepsilon|$. Let $\tilde{M} \in J_{n-p}$. We note that if there is an $M \in J_{n}$ for which $T^{-p} M \subset \tilde{M}$, and $k_{\sigma(M)}=p$, we have $\tilde{M} \cap S_{0} \neq \emptyset$. For such an $M$, we have

$$
l\left(T^{-p}\left(A_{\varepsilon} \cap M\right)\right) \leqq \lambda^{-p}, \quad l\left(A_{\varepsilon} \cap M\right)<4 \varepsilon \lambda^{-p},
$$

and from $l(\tilde{M})>4 \varepsilon^{\varrho} \lambda^{-p}\left[\right.$ since $\left.p=k_{\sigma(M)}(M)\right]$, we deduce

$$
\begin{aligned}
\sum_{\substack{M \in E_{2} \\
T^{-p} M \subset \tilde{M} \\
k_{\sigma(M)}(M)=p}} l\left(T^{-p}\left(A_{\varepsilon} \cap M\right)\right) & <4 \varepsilon \lambda^{-p} L_{p} \leqq 4 \varepsilon \lambda^{-p} 2^{1+p / K} \\
& <2 \varepsilon^{1-\varrho-\frac{c}{K} \log 2} l(\tilde{M}) .
\end{aligned}
$$

If $M \in J_{n}$ and $T^{-p} M \subset \tilde{M}, \tilde{M} \in J_{n-p}$, we have

$$
\frac{l\left(T^{-n}\left(A_{\varepsilon} \cap M\right)\right)}{l\left(T^{-n+p} \tilde{M}\right)}=\frac{l\left(T^{-p}\left(A_{\varepsilon} \cap M\right)\right)}{l(\tilde{M})} .
$$

Therefore,

and

$$
\sum_{\substack{M \in E_{2} \\ T^{-p} M \subset \tilde{M} \\ k_{\sigma(M)(M)=p}}} l\left(T^{-n}\left(A_{\varepsilon} \cap M\right)\right)<2 \varepsilon^{1-\varrho-\frac{c}{K} \log 2} l\left(T^{-(n-p)} \tilde{M}\right)
$$

$$
\begin{aligned}
\sum_{M \in E_{2}} l\left(T^{-n}\left(A_{\varepsilon} \cap M\right)\right) & =\sum_{p=0}^{E(c|\log \varepsilon|)} \sum_{\tilde{M} \in J_{n-p}} \sum_{\begin{array}{c}
M \in E_{2} \\
T^{-p} \mathcal{M} \subset \tilde{M} \\
k_{\sigma(M)}(M)=p
\end{array}} l\left(T^{-n}\left(A_{\varepsilon} \cap M\right)\right) \\
& <2 \varepsilon^{1-\varrho-\frac{c}{K} \log 2} \sum_{p=0}^{E(c|\log \varepsilon|)} \sum_{\tilde{M} \in J_{n-p}} l\left(T^{-(n-p)} \tilde{M}\right) \\
& <4 \varepsilon^{1-\varrho-\frac{c}{K} \log 2} c|\log \varepsilon| l\left(L_{0}\right) .
\end{aligned}
$$

Let now $E_{2}$ be defined by $E_{3}=J_{n} \backslash\left(E_{1} \cup E_{2}\right)$. We shall assume $E_{3} \neq \emptyset$, otherwise, the proof is finished. This implies $n>c|\log \varepsilon|$. For $M \in E_{3}$, let $i$ be the unique integer 
such that

$$
k_{i}(M)<c|\log \varepsilon| \leqq k_{i+1}(M) .
$$

Note that $k_{i+1}(M)$ exists if $\varepsilon_{0}$ is small enough since $l\left(L_{0}\right)>1$. We now observe that $T^{-\left(k_{i+1}-k_{i}\right)} R_{k_{\imath}}(M) \cap S_{0}^{-} \neq \emptyset$, and

$$
l\left(T^{-\left(k_{\imath+1}-k_{i}\right)} R_{k_{i}}(M)\right)<\lambda^{-\left(k_{i+1}-k_{i}\right)} l\left(R_{k_{\imath}}(M)\right) \leqq 4 \varepsilon^{\varrho} \lambda^{-k_{i+1}} .
$$

Therefore, $T^{-\left(k_{i+1}-k_{i}\right)} R_{k_{i}}(M) \subset A_{4 \varepsilon^{\varrho} \lambda^{-k_{1+1}}}$. We shall now use the recursive assumption. We have

$$
\begin{aligned}
\sum_{\substack{M \in E_{3} \\
k_{2}+1(M)=p}} l\left(T^{-n}\left(A_{\varepsilon} \cap M\right)\right) & =\mu_{n-p}^{\prime}\left(\bigcup_{\substack{M \in E_{3} \\
k_{i}+1(M)=p}} T^{-p}\left(A_{\varepsilon} \cap M\right)\right) \cdot l\left(L_{0}\right) \\
& \leqq \mu_{n-p}^{\prime}\left(A_{4 \varepsilon^{\varrho} \lambda-p}\right) \cdot l\left(L_{0}\right) .
\end{aligned}
$$

Therefore,

$$
\begin{aligned}
\sum_{M \in E_{3}} l\left(T^{-n}\left(A_{\varepsilon} \cap M\right)\right) & \leqq \sum_{p=c|\log \varepsilon|}^{n} \mu_{n-p}^{\prime}\left(A_{4 \varepsilon \varrho \lambda-p}\right) \\
& \leqq \frac{4^{\tau} \varepsilon^{\varrho \tau} \lambda^{-c \tau|\log \varepsilon|}}{1-\lambda^{-\tau}}
\end{aligned}
$$

From $\varrho+c \log \lambda>1,1-\varrho>\tau$, and $1-\varrho-\frac{c}{K} \log 2>\tau$, we obtain $\mu_{n}^{\prime}\left(A_{\varepsilon}\right) \leqq \varepsilon^{\tau}$ if $\varepsilon<\varepsilon_{0}$ for $\varepsilon_{0}$ small enough but independent of $n$. Q.E.D.

We fix now $\tau$ as in Proposition III.1 and denote by $\left(\mu_{n}\right)_{n \in \mathbb{N}}$ a subsequence of the previous sequence which converges weakly to $\mu$.

Corollary III.3. i) For $\varepsilon$ small, $n \in \mathbb{N}, k \in \mathbb{Z}, \mu_{n}\left(T^{k} A_{\varepsilon}\right)<\varepsilon^{\tau}, \mu\left(T^{k} A_{\varepsilon}\right)<\varepsilon^{\tau}$.

ii) For $N \in \mathbb{N}$, we define $H^{N}=\left\{x \in \Omega\right.$ : one (at least) of the endpoints of $W_{\mathrm{loc}}^{u}(x)$ does not lie on $\left.\bigcup_{k=0}^{N} S_{k}^{+}\right\}$. There exist $c>0$ and $\alpha, 0<\alpha<1$, such that for $n \in \mathbb{N}$, $\mu_{n}\left(H^{N}\right)<c \alpha^{N}$, and $\mu\left(H^{N}\right)<c \alpha^{N}$.

iii) For $\varepsilon \in \mathbb{R}_{*}^{+}$, we define $H_{\varepsilon}=\{x \in \Omega$ : one (at least) of the semi-loc unstable manifolds is shorter than $\varepsilon\}$. For $\varepsilon$ small, $\mu_{n}\left(H_{\varepsilon}\right)<\varepsilon^{\tau}, n \in \mathbb{N}$, and $\mu\left(H_{\varepsilon}\right)<\varepsilon^{\tau}$.

iv) For $x \in \Omega, \mu(\{x\})=0$.

v) For $x \in \Omega, \mu\left(\left\{W^{u}(x)\right\}\right)=\mu\left(\left\{W^{s}(x)\right\}\right)=0$.

Proof. The first part of i) follows from Proposition III.1, by the definition of $\mu_{n}$; the second part is a consequence of the weak convergence of $\left(\mu_{n}\right)$, for $A_{\varepsilon}$ has smooth boundary.

To prove ii) consider the line $\Delta$ passing through $x \in \Omega$ in the unstable direction, and let $y$ be one of the endpoints of $W_{\text {loc }}^{u}(x)$.

By construction, either $y \in S_{k}^{+}$for some $k \in \mathbb{N}$ or $y \notin \bigcup_{k \geqq 0} S_{k}^{+}$, but $y$ is an accumulation point of $\bigcup_{k \geqq 0} S_{k}^{+} \cap \Delta$. In the latter case, one can construct a sequence $\left(y_{n}\right)_{n \in \mathbb{N}}, y_{n} \in \Delta$, such that, for some strictly increasing function $k(\cdot), y_{n} \in S_{k(n)}^{+}, n \in \mathbb{N}$, 
and moreover, $\bigcup_{k=0}^{k(n)} S_{k}^{+}$does not cross $\Delta$ between $y_{n}$ and $x$. Then, $T^{-k(n)}$ is linear on the segment $\left[x, y_{n}\right] \subset \Delta$. As $\lim _{n \rightarrow \infty} y_{n}=y \in \Omega$ and $\Omega$ is bounded, we can suppose $d\left(x, y_{n}\right)<10$, so that $d\left(T^{-k(n)} x, S_{0}^{+}\right)<10 / \lambda^{k(n)}, \forall n \in \mathbb{N}$. Thus, $x \in \bigcap_{n \geqq 0} T^{k(n)} A_{10 / \lambda^{k(n)}}$, which is a set of $\mu$-measure zero, by i): almost surely, the endpoints of $W_{\text {loc }}^{u}(x)$, $x \in \Omega$, lie in $\bigcup_{k \geqq 0} S_{k}^{+}$. Suppose now $y \in S_{k}^{+}, k \geqq N$. Then $x \in T^{k} A_{10 / \lambda^{k}}$ whence ii) by i) with $\alpha=\lambda^{-\tau}$.

With the same notations, suppose $x \in H_{\varepsilon}$; then if $y \in S_{k}^{+}, k \in \mathbb{N}, x \in T^{k} A_{\varepsilon / \lambda^{k}}$, whence iii) by i) and the convergence of $\sum_{k \geqq 0} \lambda^{-k \tau}$.

The proof of iv) is similar to the proof of Proposition III.1: Let $x \in \Omega$, and let $\varepsilon$ be a positive number. Let $B$ be the ball of radius $\varepsilon$ centered at $x$. Let $J_{n}$ be as above. We have

$$
\mu_{n}(B)=\sum_{M \in J_{n}} l\left(T^{-n}(B \cap M)\right) / l\left(L_{0}\right) .
$$

Let $J_{n}^{+}=\left\{M \in J_{n} \mid l(M)>\varepsilon^{\frac{1}{1+\tau}}\right\}, J_{n}^{-}=J_{n} \backslash J_{n}^{+}$. If $M \in J_{n}^{+}$, from $l(B \cap M) \leqq 2 \varepsilon$, we obtain

$$
l\left(T^{-n}(B \cap M)\right)=l\left(T^{-n} M\right) \frac{l(B \cap M)}{l(M)} \leqq \varepsilon^{\frac{\tau}{1+\tau}} l\left(T^{-n} M\right)
$$

Therefore,

$$
\sum_{M \in J_{n}^{+}} l\left(T^{-n}(B \cap M)\right) \leqq \varepsilon^{\frac{\tau}{1+\tau}} \sum_{M \in J_{n}^{+}} l\left(T^{-n} M\right) \leqq \varepsilon^{\frac{\tau}{1+\tau}} l\left(L_{0}\right)
$$

For $J_{n}^{-}$, we have

$$
\begin{aligned}
\sum_{M \in J_{n}^{-}} l\left(T^{-n}(B \cap M)\right) & \leqq \sum_{M \in J_{\bar{n}}^{-}} l\left(T^{-n} M\right)=\mu_{n}\left(\bigcup_{M \in J_{\bar{n}}^{-}} M\right) \cdot l\left(L_{0}\right) \\
& \left.\leqq \mu_{n}\left(H \varepsilon^{1 / 1+\tau}\right) \cdot l\left(L_{0}\right) \leqq c \cdot l\left(L_{0}\right) \cdot \varepsilon^{\frac{\tau}{1+\tau}} \quad \text { by iii }\right)
\end{aligned}
$$

We obtain

$$
\mu(B) \leqq l\left(L_{0}\right)(1+c) \cdot \varepsilon^{\frac{\tau}{1+\tau}}
$$

and get iv) if we let $\varepsilon \rightarrow 0$.

We shall prove v) for $W^{s}(x)$, the proof for $W^{u}(x)$ is similar. It is enough to prove that $\left(W_{\mathrm{loc}}^{s}(x)\right)=0$, since $W^{s}(x)=\bigcup_{j=0}^{\infty} T^{-j} W_{\mathrm{loc}}^{s}\left(T^{j} x\right)$. Assume $x \in \Omega$ satisfies $\mu\left(W_{\mathrm{loc}}^{\mathrm{s}}(x)\right)>\beta>0$. Then, there is a couple of integers $i$ and $j, i \neq j$ such that $T^{i} W_{\mathrm{loc}}^{\mathrm{s}}(x)$ $\cap T^{j} W_{\mathrm{loc}}^{s}(x) \neq \emptyset$, otherwise, we get a contradiction from $\mu\left(T^{l} W_{\mathrm{loc}}^{s}(x)\right)>\beta, \forall l \geqq 0$. Assume $i>j$ and let $k=i-j$. Since $T$ is a bijection, we have $T^{k} W_{\mathrm{loc}}^{s}(x) \cap W_{\mathrm{loc}}^{s}(x) \neq \emptyset$, and therefore, $T^{k} W_{\mathrm{loc}}^{s}(x) \subset W_{\mathrm{loc}}^{s}(x)$. Since $\left.T^{k}\right|_{W_{\mathrm{loc}}^{s}(x)}$ is a contraction, this means that $W_{\mathrm{loc}}^{s}(x)$ contains a $k$ periodic point $P$. Moreover, $P=\bigcap_{l=0}^{\infty} T^{k l} W_{\mathrm{loc}}^{s}(x)$, and
therefore,

$$
\mu(\{P\})=\inf _{l} \mu\left(T^{k l} W_{\mathrm{loc}}^{s}(x)\right)=\mu\left(W_{\mathrm{loc}}^{s}(x)\right)>\beta .
$$

a contradiction with iv). 


\section{Ergodic Properties of the Invariant Measure}

So far, the measure $\mu$ is not unique. The uniqueness will be proven by showing that $\mu$ is the Bowen-Ruelle measure. We first investigate the properties of the conditional expectations of $\mu$ on the unstable foliation, making use of the sequence $\left(\mu_{n}\right)_{n \in \mathbb{N}}$. In order to investigate and use these properties, we define two countable partitions $\alpha$ and $\beta$ which decompose $\Omega$.

Let $\zeta^{+}\left(\zeta^{-}\right)$be the decomposition of $\mu$.a.a. $\Omega$ into local unstable (stable) manifolds. As $\zeta^{+}$is a partition generated by $\bigcup_{n \geq 0} S_{n}^{+}$, it is measurable. We can define the restriction $\mu^{+}$of $\mu$ to the sub $\sigma$ algebra $\mathscr{M}^{+} C \mathscr{M}_{\Omega}$ of the sets $\zeta^{+}$-saturate. By Corollary III.3ii), for $\mu$.a.e. $W_{0} \in \zeta^{+}$, there are two maximal smooth components $I_{0}$ and $J_{0}$, contained in $\bigcup_{k=0}^{N_{0}} S_{k}^{+}$for some $N_{0} \geqq 0$ such that the endpoints of $W_{0}$ lie on $I_{0}$ and $J_{0}$. We then define the partition $\alpha$ by $\alpha\left(W_{0}\right)=\left\{W \in \zeta^{+}\right.$with endpoints on $I_{0}$ and $\left.J_{0}\right\}$. Let $\zeta_{N}^{+} \subset \zeta^{+}$be the union of the elements of $\zeta^{+}$with endpoints on $\bigcup_{k=0}^{N} S_{k}^{+}$, $N \in \mathbb{N}$. $\zeta_{N}^{+}$is a finite union of atoms of $\alpha$; since, by Corollary III.3ii), $\lim _{N \rightarrow \infty} \mu^{+}\left(\zeta_{N}^{+}\right)=1, \alpha$ is a countable partition of $\zeta^{+}$.

We now look for a partition of $\Omega$ into parallelograms. Let $P_{N}^{+}\left(P_{N}^{-}\right)$be the partitions generated by $\bigcup_{n=0}^{N} S_{n}^{+}\left(\bigcup_{n=0}^{N} S_{n}^{-}\right)$. As $S_{n}^{+}$is a broken line, folded only on $\bigcup_{i=0}^{n-1} S_{i}^{+}$, a simple recursion argument shows that the $P_{N}^{ \pm}(x), x \in \Omega$, are convex sets. Note that $\zeta^{ \pm}=\lim _{N \rightarrow \infty} P_{N}^{ \pm}$. Let $x \in \Omega$ such that $W^{+}=W_{\mathrm{loc}}^{u}(x)$ and $W^{-}=W_{\mathrm{loc}}^{s}(x)$ have positive length. Let $N_{ \pm}(x)$ be the smallest integers such that the endpoints of $W^{-}$ $\left(W^{+}\right)$lie outside $P_{N^{+}(x)}^{+}(x)\left[P_{N^{-}(x)}^{-}(x)\right]$. It is easy to see that $N_{ \pm}$are finite; $N_{ \pm}$are obviously measurable functions, so that we can define, for such an element $x \in \Omega$ the atom $\beta(x)$ by:

$$
\beta(x)=N_{x}^{-1}\left(N_{+}(x)\right) \cap N_{-}^{-1}\left(N_{-}(x)\right) \cap P_{N^{+}(x)}^{+}(x) \cap P_{N^{-}(x)}^{-}(x) .
$$

As usual, we define the unstable and stable fibers $\gamma^{+}(x), \gamma^{-}(x)$ by

$$
\begin{gathered}
\gamma^{+}(x)=\beta(x) \cap W_{\mathrm{loc}}^{u}(x), \\
\gamma^{-}(x)=\beta(x) \cap W_{\mathrm{loc}}^{s}(x), \quad \text { i.e. } \quad \gamma^{ \pm}=\beta \vee \zeta^{ \pm} .
\end{gathered}
$$

The set $\beta(y)$ is a parallelogram in the following sense

$$
x \in \beta(y) \Rightarrow \begin{cases}\exists ! z \in \beta(y), & z=\gamma^{+}(x) \cap \gamma^{-}(y), \\ \exists ! z^{\prime} \in \beta(y), & z^{\prime}=\gamma^{-}(x) \cap \gamma^{+}(y) .\end{cases}
$$

This is easily checked by using the convexity of $P_{N^{ \pm}(x)}^{ \pm}(x)$ and the fact that $N^{ \pm}$are constant on atoms of $P_{N^{ \pm}(x)}^{ \pm} \vee \zeta^{\mp}$. By definition $\beta$ is a countable partition of $\Omega$ into parallelograms.

We now come back to the properties of $\mu$. As $\zeta^{+}$is a measurable partition, we can apply the usual theorem on disintegration (see [Ro] e.g.).

Namely, there is a $\mu$-a.s. unique family $\left\{\mu_{W}, W \in \zeta^{+}\right\}$of probability measures on $\Omega$ such that: 
a) $\mu_{W}$ has $\mu$-a.s. support on $W$,

b) for $A \in \mathscr{M}_{\Omega}$ the map $W \rightarrow \mu_{W}(A)$ is in $L^{1}\left(\zeta^{+}, d \mu^{+}\right)$, and $\mu(A)$ $=\int_{\zeta^{+}} \mu_{W}(A) d \mu^{+}(W)$, denoted $\mu^{+}(\mu .(A))$.

For $W \in \zeta^{+}$, let $l_{W}$ denote the normalized $1-d$ Lebesgue measure on $W$.

Proposition IV.1. The conditional expectations of $\mu$ on the local unstable manifolds are the corresponding $1-d$ Lebesgue probabilities, i.e.: $\mu_{W}=l_{W}$ for $\mu$.a.e. $W \in \zeta^{+}$.

Proof. As stated before, some subsequence $\left(\mu_{n_{i}}\right)_{i \in \mathbb{N}}$ of $\left(\mu_{n}\right)_{n \in \mathbb{N}}$ converges weakly to $\mu$; we shall still denote it $\left(\mu_{n}\right)_{n \in \mathbb{N}}$. Let $\mu_{n}^{+}$denote the restriction of $\mu_{n}$ to $\mathscr{M}^{+}, n \in \mathbb{N}$. By the geometrical properties of $T$, it is easy to show that for $A \in \mathscr{M}_{\Omega}, \mu_{n}(A)$ $=\int_{\zeta^{+}} l_{W}(A) d \mu_{n}^{+}(W)=\mu_{n}^{+}(l .(A))$. It is enough to prove $\lim _{n \rightarrow \infty} \mu_{n}^{+}(l .(f))=\mu^{+}(l .(f))$ for $f \in C^{0}(\Omega)$. Let $f \in C^{0}(\Omega)$. With respect to the Hausdorff topology $\tau_{H}$ on $\zeta^{+}$, the map $\zeta^{+} \rightarrow \mathbb{R}: W \rightarrow l_{W}(f)$ is continuous. We shall show that $\mu_{n}^{+}$converges weakly to $\mu^{+}$in the sense of $\tau_{H}$.

By Corollary III.3ii), $\mu_{k}^{+}\left(\zeta^{+} \backslash \zeta_{N}^{+}\right), k \in \mathbb{N}$ and $\mu^{+}\left(\zeta^{+} \backslash \zeta_{N}^{+}\right)$are simultaneously bounded by $C \cdot \varrho^{N}$ for some $\left.\varrho \in\right] 0,1$ [ and some positive constant $C$. Let $Q \subset \zeta^{+}$be an atom of $\alpha$. Since $\zeta_{N}^{+}$is a finite union of atoms of $\alpha$ the compactness of $\zeta_{N}^{+}$would follow from the compactness of $Q$. As two elements of $\zeta^{+}$cannot intersect, the elements of $Q$ depend continuously on, e.g. the vertical coordinate of their rightmost endpoint. Since $\Omega$ is compact, the limit of a convergent sequence of elements of $Q$ is a segment contained in $\Omega$, thus, it belongs to $Q: Q$ is compact. As $\Omega$ is totally regular, the hypothesis of Prokhorov's theorem are fulfilled (cf. [B]), so that $\left(\mu_{n}^{+}\right)_{n \in \mathbb{N}}$ converges weakly to some probability measure on $\zeta^{+}$, which has to be the restriction $\mu^{+}$of $\mu$. Q.E.D.

We are now able to derive the ergodic properties of $T$.

Proposition IV.2. $(\Omega, T, \mu)$ is ergodic.

Proof. Take $f \in C^{0}(\Omega)$. By Birkhoff's ergodic theorem, there is a set $B \subset \Omega, \mu(B)=0$ and a function $\bar{f} \in L^{1}(d \mu)$ such that, if $x \in \Omega \backslash B$ the limits $f^{ \pm}(x)$ $=\lim _{N \rightarrow \infty} \frac{1}{N} \sum_{n=0}^{N-1} f\left(T^{ \pm n} x\right)$ exist and $f^{+}(x)=f^{-}(x)=\bar{f}(x)$. What we shall prove is that $\bar{f}$ is almost surely constant. Let $x, y \in \Omega \backslash B$. As $\mu(B)=\mu\left(\bigcap_{\varepsilon} H_{\varepsilon}\right)=0$, the conditional measures of $B \cup\left(\bigcap_{\varepsilon} H_{\varepsilon}\right)$ (i.e. the corresponding normalized lengths) are zero on $W_{\text {loc }}^{u}(x)$ and $W_{\text {loc }}^{u}(y)$ for $\mu \times \mu$-a.e. $(x, y)$. Thus, by Proposition II.4, it is enough to find a subset $A \subset W_{\text {loc }}^{u}(x)$ of positive length and an integer $N$ such that for $z \in A, W_{\mathrm{loc}}^{\mathrm{s}}(z)$ crosses $T^{N} W_{\mathrm{loc}}^{n}(y)$, because $f^{+}\left(f^{-}\right)$is obviously constant on stable (unstable) manifolds. Consider now $\beta(x)$. Almost surely $\mu(\beta(x))>0$, and thus, by Proposition II.4, $l\left(\gamma^{+}(x)\right)>0$. By Corollary III.3v), we can define $x_{1}, x_{2} \in \beta(x)$ by demanding that the quadrilateral $Q$ defined by $\zeta^{ \pm}\left(x_{1}\right), \zeta^{ \pm}\left(x_{2}\right)$ be the smallest such that $\mu(Q \cap \beta(x))=\mu(\beta(x))$.

For some $N$, by Proposition II.3, $T^{N} W_{\text {loc }}^{u}(y)$ "enters" $Q$, and one of its smooth components, that we shall call $\tilde{W}$, crosses $\zeta^{-}\left(x_{1}\right)$ or $\zeta^{-}\left(x_{2}\right)$ or both. Thus, we can 
define the canonical isomorphism $P$ along stable fibers from $W_{\text {loc }}^{u}(x)$ into $\tilde{W} \subset T^{N} W_{\text {loc }}^{u}(y)$, with a domain $\mathscr{D}(P)$ of positive length. The proof is completed by taking $A=\mathscr{D}(P)$.

Proposition IV.3. i) For $n \in \mathbb{N}^{*},\left(\Omega, T^{n}, \mu\right)$ is ergodic.

ii) $(\Omega, T, \mu)$ is a $K$-system.

Proof. The proof of i) is similar to the proof of Proposition IV.2 ii) follows from the fact the Pinsker $\sigma$-algebra $\Pi$ of $T$ is smaller than the $\sigma$-algebra of the measurable sets saturate by stable and unstable manifolds (cf. [P]). The proof of Proposition IV.2 shows that if $f \in L^{1}(d \mu)$ is constant along stable and unstable manifolds then $f$ is $\mu$-a.s. constant; thus, $\Pi$ is trivial for $\mu$, whence ii).

In order to prove the Bernoullian property, we introduce some notations. We define decreasing sequences $\left(\mathscr{M}_{n}^{ \pm}\right)_{n \in \mathbb{N}}$, where $\mathscr{M}_{n}^{ \pm}$is the sub $\sigma$-algebra of the elements of $\mathscr{M}_{\Omega}$ which are $T^{ \pm n} \zeta^{ \pm}$-saturate. By Proposition IV.3ii) the $\sigma$-algebras $\lim _{n} \mathscr{M}_{n}^{ \pm}=\bigcap_{n} \mathscr{M}_{n}^{ \pm}$are both trivial for $\mu$. We note $\mu_{ \pm}$the restriction of $\mu$ to $\mathscr{M}_{0}^{ \pm}$.

Proposition IV.1 about the conditional expectation of $\mu$ with respect to $\mathscr{M}_{0}^{+}$ will allow us to prove the following:

Proposition IV.4. $(\Omega, T, \mu)$ is isomorphic to a Bernoulli shift.

Proof. The proof is similar to [L1].

The canonical map $\left(\Omega, \mathscr{M}_{0}^{+} \vee \mathscr{M}_{0}^{-}\right) \stackrel{P}{\rightarrow}\left(\zeta^{+} \times \zeta^{-}, \mathscr{M}_{0}^{+} \times \mathscr{M}_{0}^{-}\right)$given by $P(x)$ $=\left(\zeta^{+}(x), \zeta^{-}(x)\right)$ is a.e. defined. Let $\nu=\mu \circ P^{-1}$ denote the image of $\mu$ through $P$. We have:

Lemma IV.5. $v$ is absolutely continuous with respect to $\mu^{+} \otimes \mu^{-}$, i.e. $v \ll \mu^{+} \otimes \mu^{-}$. Proof. Let $A \in \mathscr{M}_{0}^{+} \vee \mathscr{M}_{0}^{-}$such that $P(A) \in \mathscr{M}_{0}^{+} \times \mathscr{M}_{0}^{-}$. By definition, we have:

$$
\mu^{+} \otimes \mu^{-}(P(A))=\int_{W \in \zeta^{+}} d \mu^{+}(W) \cdot \mu^{-}\left(\bigcup_{x \in A \cap W} \zeta^{-}(x)\right) .
$$

Suppose $\mu^{+} \otimes \mu^{-}(P(A))=0$. Then, for some $\mathscr{W}_{1} \subset \zeta^{+}$of full measure:

$$
W \in \mathscr{W}_{1} \Rightarrow \mu^{-}\left(\bigcup_{x \in A \cap W} \zeta^{-}(x)\right)=0 .
$$

Suppose that, for some $W^{\prime} \in \gamma^{+}$, included in an element $C$ of $\beta$ of positive measure, we have $\mu\left(A \mid W^{\prime}\right)=0$. Then, by Proposition II.4 and Proposition IV.1, we have:

$$
\mu\left(C \cap \bigcup_{x \in A \cap W^{\prime}} \zeta^{-}(x)\right)>0
$$

so that if $W$ is the $\zeta^{+}$-saturate of $W^{\prime}$, we have:

$$
\mu^{-}\left(\bigcup_{x \in A \cap W} \zeta^{-}(x)\right)>0
$$

so that $W \notin \mathscr{W}_{1}$. Thus, $\mu\left(A \mid W^{\prime}\right)=0$ almost surely and $\mu(A)=0$. Q.E.D.

We shall denote the measure $\mu^{+} \otimes \mu^{-}$by $\mu^{\times}$. By Radon-Nikodym's theorem, there is a $\mu^{\times}$-integrable function $h: \zeta^{+} \times \zeta^{-} \rightarrow \mathbb{R}^{+}$, such that $d v(x)=h(x) \cdot d \mu^{\times}(x)$. If $A \in \mathscr{M}_{0}^{+} \times \mathscr{M}_{0}^{-}$and $v(A)>0$, the conditional probability $v_{A}=v(\cdot \mid A)$ is given, for 
a function $f$, by:

$$
\begin{aligned}
v_{A}(f) & =v\left(f \cdot \chi_{A}\right) / v\left(\chi_{A}\right)=\mu^{\times}\left(f \cdot h \cdot \chi_{A}\right) / \mu^{\times}\left(h \cdot \chi_{A}\right) \\
& =\mu^{\times}(f \cdot h) / \mu_{A}^{\times}(h)
\end{aligned}
$$

or, more briefly:

$$
v_{A}=\mu_{A}^{\times}(h \cdot) / \mu_{A}^{\times}(h) .
$$

We are now able to prove the weak Bernoulli property.

Lemma IV.5. $(\Omega, \mathscr{M}, \mu, T)$ is weak Bernoulli, that is $\mu$ and $\mu^{\times} \circ P$ coincide on $\bigwedge_{n}\left(\mathscr{M}_{n}^{+} \vee \mathscr{M}_{n}^{-}\right)$

Proof. As $\left(\mathscr{M}_{n}^{+} \vee \mathscr{M}_{n}^{-}\right)_{n \in \mathbb{N}}$ is a decreasing sequence, the conditional expectation on $A$ of $\mu$ with respect to $\bigwedge_{n}\left(\mathscr{M}_{n}^{+} \vee \mathscr{M}_{n}^{-}\right)$is given almost everywhere by:

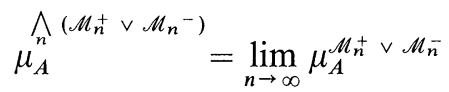

$$
\begin{aligned}
& =\lim _{n \rightarrow \infty} v_{P(A)}^{\mathscr{M}_{n}^{+} \times \mathscr{M}_{n}^{-}},
\end{aligned}
$$

by definition of $v$. Therefore,

$$
\bigwedge_{n}^{\bigwedge_{n}\left(\mathscr{M}_{n}^{+} \vee \mathscr{M}_{n}^{-}\right)}=\lim _{n \rightarrow \infty}\left(\mu^{\times}\right)_{P(A)}^{\mathscr{M}_{n}^{+} \times \mathscr{M}_{n}^{-}}(h \cdot) /\left(\mu^{\times}\right)_{P(A)}^{\mathscr{M}_{n}^{+} \times \mathscr{M}_{n}^{-}}(h) .
$$

As $\left(\Omega, \mathscr{M}_{\Omega}, \mu, T\right)$ is a $K$-system, the $\sigma$-algebras $\bigwedge_{n \in \mathbb{N}} \mathscr{M}_{n}^{+}$and $\bigwedge_{n \in \mathbb{N}} \mathscr{M}_{n}^{-}$both coïncide with the trivial algebra $\bmod \mu$ : the conditional expectations

$$
\mu^{\widehat{\Lambda}_{n \in \mathbb{N}} \mu_{n}^{ \pm}}=\lim _{n \rightarrow \infty} \mu_{ \pm}^{\mathscr{M}_{n}^{ \pm}}
$$

are constant $\mu$-almost everywhere.

Thus, both $\mu^{\wedge\left(\mathscr{M}_{n}^{+} \vee \mathscr{M}_{n}^{-}\right)}$and $\left(\mu^{\times} \circ P\right)^{\bigwedge_{n}\left(\mathscr{M}_{n}^{+} \vee \mathscr{M}_{\bar{n}}^{-}\right)}$are $\mu$-a.e. constant and thus coïncide. Q.E.D.

The statement of Proposition IV.4 follows from Lemma IV.5 (cf. [L2] for instance).

We prove now that $\mu$ is the (unique) Bowen-Ruelle measure, id est:

Proposition IV.6. For $g \in C^{0}(F)$ and $m$-almost any $x \in F$,

$$
\lim _{n \rightarrow \infty} \frac{1}{n} \sum_{k=0}^{n-1} g\left(T^{k} x\right)=\mu(g) \text {. }
$$

The proof follows three steps: We consider the points $x$ of $\bigcup_{n \in \mathbb{N}} T^{n} L_{0}$ such that, for some $g \in C^{0}(F)$, we have not $\lim _{N \rightarrow \infty} \frac{1}{N} \sum_{n=0}^{N-1} g\left(T^{n} x\right)=\mu(g)$, and prove that the length of this set is zero (Lemma IV.7). Then we notice that, for $m$-a.e. $x \in F$ one can find positive integers $p, n$ such that $W_{\text {loc }}^{s}\left(T^{n} x\right)$ crosses $T^{p} L_{0}$ (Lemma IV.8). Thus, 
what we have to show is that, $m$-almost surely in $F$, this intersection does not fall in the exceptional set estimated in Lemma IV.7.

Let $\mathscr{A}=\left\{x \in \bigcup_{n \geqq 0} T^{n} L_{0} \mid \exists g \in C^{0}(F)\right.$ and $\lim _{N \rightarrow+\infty} \frac{1}{N} \sum_{n=0}^{N-1} g\left(T^{n} x\right)$ does not exist or is not equal to $\mu(g)\}$.

Lemma IV.7. $\forall n \in \mathbb{N}, \mu_{n}(\mathscr{A})=l\left(\mathscr{A} \cap L_{0}\right)=0$.

Proof. We first observe that $\mathscr{A}=T \mathscr{A}$, and if $x, y \in T^{n} L_{0}, y \in W_{\mathrm{loc}}^{s}(x)$, then $x \in \mathscr{A}$ is equivalent to $y \in \mathscr{A}$. This last property implies using Propositions II.4 and IV.1, that $W \rightarrow \mu(\mathscr{A} \mid W)$ is a continuous function on $\left(\zeta^{+}, \tau_{H}\right)$. From $\mu\left(\bigcup_{n \geqq 0} T^{n} L_{0}\right)=0$, we deduce $\mu(\mathscr{A})=0$. Therefore, using the Birkhoff ergodic Theorem, we have

$$
0=\mu(\mathscr{A})=\int_{\zeta^{+}} d v(W) \mu(\mathscr{A} \mid W)=\lim _{n \rightarrow+\infty} \int_{\zeta^{+}} d v_{n}(W) \mu(\mathscr{A} \mid W)=\lim _{n \rightarrow+\infty} \mu_{n}(\mathscr{A}) .
$$

However, $\mu_{n}(\mathscr{A})=\frac{l\left(T^{-n} \mathscr{A} \cap L_{0}\right)}{l\left(L_{0}\right)}=\frac{l\left(\mathscr{A} \cap L_{0}\right)}{l\left(L_{0}\right)}$, hence $l\left(\mathscr{A} \cap L_{0}\right)=0 . \quad$ Q.E.D.

Let $\mathscr{L}=\left\{x \in F \mid \forall n, p \in \mathbb{N}, W_{\mathrm{loc}}^{s}\left(T^{n} x\right) \cap T^{p} L_{0}=\emptyset\right\}$.

Lemma IV.8. $m(\mathscr{L})=0$.

Proof. Let $H$ and $r$ be as in Lemma II.2. This lemma implies $\mathscr{L} \subset \bigcup_{n>0} T^{-n}(\mathscr{L} \cap H)$. Therefore, it is enough to show that $m(\mathscr{L} \cap H)=0$ since $T^{-1}$ is absolutely continuous. For $\alpha>0$ and $n \in \mathbb{N}$, let $\mathscr{M}_{n}^{\alpha}=\left\{x \in H \mid l\left(W_{\text {loc }}^{s}\left(T^{n} x\right)\right)<\alpha^{n}\right\}$. We have

$$
\mathscr{M}_{n}^{\alpha}=T^{-n}\left\{y \in T^{n} H \mid l\left(W_{\mathrm{loc}}^{s}(y)\right)<\alpha^{n}\right\}
$$

and using $[\mathrm{M}]$, we derive

$$
m\left(\mathscr{M}_{n}^{\alpha}\right) \leqq \delta\left(\frac{\alpha}{b}\right)^{n}
$$

where $\delta$ is a positive constant. Therefore, if $\alpha<b$, we have with $\mathscr{M}^{\alpha}=\bigcap_{n=0}^{\infty} \mathscr{M}_{n}^{\alpha}$,

$$
m\left(\mathscr{M}^{\alpha}\right)=0 .
$$

Let $x \in\left(H \backslash \mathscr{M}^{\alpha}\right) \cap \mathscr{L}$. We have $x \notin \mathscr{M}_{n}^{\alpha}$ for $n$ large enough, but

$$
W_{\mathrm{loc}}^{s}\left(T^{n} x\right) \cap T^{n+r} L_{0}=\emptyset \text {, }
$$

therefore,

$$
W_{\mathrm{loc}}^{s}\left(T^{n} x\right) \subset T^{n} H
$$

$T^{-n} W_{\mathrm{loc}}^{s}\left(T^{n} x\right)$ is a broken line of total length bounded below by $\left(\frac{\lambda \alpha}{b}\right)^{n}$. However, for $b$ small enough, it is easy to verify that for $y \in H$ such that $W_{\text {loc }}^{s}(y) \subset H$, then $W_{\text {loc }}^{s}(y)$ does not intersect both $S_{0}^{-}$and $S_{0}^{+}$. This implies that $T^{-n} W_{\text {loc }}^{s}\left(T^{n} x\right)$ is 
composed of at most $2^{[n / 2]+1}$ straight segments. Therefore, since each segment has a length at most one, we have

$$
l\left(T^{-n}\left(W_{\mathrm{loc}}^{s}\left(T^{n} x\right)\right)\right) \leqq 2^{[n / 2]+1} .
$$

We now choose $\alpha<b$, such that $\frac{\sqrt{2}}{\lambda} \frac{b}{\alpha}<1$ (this is possible since $\lambda>\sqrt{2}$ ). We have

$$
2^{[n / 2]+1} \geqq l\left(T^{-n}\left(W_{\mathrm{loc}}^{s}\left(T^{n} x\right)\right)\right) \geqq\left(\frac{\lambda \alpha}{b}\right)^{n},
$$

which is a contradiction if $n$ is large enough. Therefore, $\mathscr{L} \cap H \subset \mathscr{M}^{\alpha}$, and we have

$$
m(\mathscr{L} \cap H) \leqq m\left(\mathscr{M}^{\alpha}\right)=0 \text {. Q.E.D. }
$$

We now come to the proof of Proposition IV.6.

We first observe that if $x \in F \backslash \mathscr{L}$, one can find an integer $n$, and $p \geqq n$ such that $W_{\text {loc }}^{s}\left(T^{n} x\right) \cap T^{p} L_{0} \neq \emptyset$. We define a new set $\mathscr{B}$ by

$$
\mathscr{B}=\left\{x \in F \backslash \mathscr{L} \mid \forall n, p, p \geqq n, W_{\text {loc }}^{s}\left(T^{n} x\right) \cap T^{p} L_{0} \subset \mathscr{A}\right\} .
$$

If $x \in F \backslash(\mathscr{L} \cup \mathscr{B})$, there is an $n \in \mathbb{N}$, and an integer $p \geqq n$ such that some point $y$ of $W_{\text {loc }}^{s}\left(T^{n} x\right) \cap T^{p} L_{0}$ does not belong to $\mathscr{A}$. Therefore, if $g$ belongs to $C^{0}\left(\mathbb{R}^{2}\right)$, we have

$$
\begin{aligned}
\lim _{m \rightarrow+\infty} \frac{1}{m} \sum_{j=0}^{m-1} g\left(T^{j} x\right) & =\lim _{m \rightarrow \infty} \frac{1}{m} \sum_{j=0}^{m-1} g\left(T^{j+n} x\right) \\
& =\lim _{m \rightarrow \infty} \frac{1}{m} \sum_{j=0}^{m-1} g\left(T^{j} y\right)=\mu(g) .
\end{aligned}
$$

We shall now show that $m(\mathscr{B})=0$. Let $\mathscr{B}_{p}$ be defined by

$$
\mathscr{B}_{p}=\left\{y \in F \backslash \mathscr{L} \mid W_{\mathrm{loc}}^{s}(y) \cap T^{p} L_{0} \subset \mathscr{A}\right\} .
$$

From $\mathscr{B}=\bigcap_{p>n \geqq 0} T^{-n} \mathscr{B}_{p}$, it is enough to show that $m\left(\mathscr{B}_{p}\right)=0$ for every integer $p$. From the definition of $\mathscr{B}_{p}$, we have

$$
\mathscr{B}_{p} \subset \bigcup_{x \in \mathscr{B} \cap T^{p} L_{0}}\left(W_{\mathrm{loc}}^{s}(x) \cap F\right) .
$$
Thus, an unstable segment $W$ being chosen, we consider $\mathscr{B}_{W}=\bigcup_{x \in \mathscr{A} \cap W} W_{\text {loc }}^{s}(x)$, and
prove $m\left(\mathscr{B}_{W}\right)=0$, using $l(\mathscr{A} \cap W)=0$.

Let $\mathscr{A}_{W}=\left\{x \in \mathscr{A} \cap W \mid W_{\text {loc }}^{s}(x) \neq\{x\}\right\}$. It is enough to prove $m\left(\bigcup_{x \in \mathscr{A}_{W}} W_{\mathrm{loc}}^{s}(x)\right)$ $=0$. If the $W_{\text {loc }}^{\text {s }}$ 's were depending smoothly on $x$, this would be a consequence of Fubini's theorem. Instead we use Lemma II.1. Let $\varepsilon>0$ be given sufficiently small. As $l(\mathscr{A} \cap W)=0, \mathscr{A} \cap W$ can be covered by a countable union of open disjoint intervals of total length smaller than $\varepsilon$. Consider one of these intervals, say $I$; let $\varepsilon^{\prime}<\varepsilon$ be its length. Let now $n \in \mathbb{N}^{*} ; \bigcup_{0}^{n+1} S_{k}^{-}$splits $I$ into at most $2^{n+2}$ segments; if $J$ is such a segment, by Lemma II.1, the dispersion of the angles of $\left\{W_{\mathrm{loc}}^{s}(x), x \in J\right\}$ is bounded by $2(b / \lambda)^{n}$, so we obtain

$$
m\left(\bigcup_{x \in J} W_{\mathrm{loc}}^{s}(x)\right) \leqq 4(b / \lambda)^{n}+l(J)
$$


Thus, $m\left(\bigcup_{x \in I} W_{\text {loc }}^{s}(x)\right) \leqq 16(2 b / \lambda)^{n}+l(I) \leqq 16(2 b / \lambda)^{n}+\varepsilon^{\prime}$. As $n$ is arbitrary and $\lambda>2 b$, we get

$$
m\left(\bigcup_{x \in I} W_{\mathrm{loc}}^{s}(x)\right) \leqq 2 \varepsilon^{\prime}
$$

so that

$$
m\left(\bigcup_{x \in \mathscr{A} W} W_{\mathrm{loc}}^{s}(x)\right) \leqq 2 \varepsilon
$$

which proves that $m\left(\mathscr{B}_{W}\right)=0$.

\section{The Hausdorff Dimension}

Let us denote by $\chi_{+}$and $\chi_{-}$the characteristic exponents of $(\Omega, T, \mu)$ : $\chi_{ \pm}=\lim _{n \rightarrow \pm \infty} \frac{1}{n} \log \left\|D_{x} T^{n}\right\|$ for $\mu$.a.e. $x \in \Omega$. Let $h$ denote the $\mu$-entropy of $T$. In [Y2] Young proves that, if $M$ is a compact surface, $(M, f, m)$ an ergodic $C^{2}$ dynamical system with characteristic exponents $\chi_{1} \geqq 0 \geqq \chi_{2}$, the Hausdorff dimension of $m$ is given by $H D(m)=h_{m}(f)\left(\frac{1}{\chi_{1}}-\frac{1}{\chi_{2}}\right)$, where $h_{m}(f)$ is the $m$-entropy of $f$. If the limit exists almost everywhere, $H D(m)$ is defined by $H D(m)=\lim _{\varepsilon \rightarrow 0} \log m(\mathscr{B}(x, \varepsilon)) / \log \varepsilon$, where $\mathscr{B}(x, \varepsilon)=\{y \in M \mid d(x, y)<\varepsilon\}$. As we shall show this result remains valid in our case, despite the fact that $T$ is only almost everywhere $C^{\infty}$. We have the following theorem.

Proposition V.1. For $\mu$-a.e. $x \in \Omega$,

$$
\lim _{\alpha \rightarrow 0} \log \mu(\mathscr{B}(x, \alpha)) / \log \alpha=h\left(\frac{1}{\chi_{+}}-\frac{1}{\chi_{-}}\right) .
$$

In order to prove this, we merely adapt ideas of Ledrappier [L 3] to our case. We prove separately two inequalities which lead to Proposition V.1.

Lemma V.2. $\lim _{\alpha \rightarrow 0} \inf \log m(\mathscr{B}(x, \alpha)) / \log \alpha \geqq h\left(\frac{1}{\chi_{+}}-\frac{1}{\chi_{-}}\right) \mu$-a.e.

Proof. Let $P$ denote the partition of $\Omega$ defined by $S=S_{0}^{+} \cup S_{0}^{-}$. For simplicity, let $F_{ \pm}(x)$ denote the quantities $\left\|D_{x} T^{ \pm 1}\right\|$, for $x \in \Omega$. Note that $F_{ \pm}$is constant on each side of $S_{0}^{\mp}$.

We first prove that, $\varepsilon>0$ being given, we can find for $\mu$-a.e. $x \in \Omega$ an integer $N(x)$ and a number $C(x, \varepsilon)>0$ such that if $n>N(x)$ and $d(x, y) \leqq C(x, \varepsilon) \cdot e^{-n}$, then $x$ and $y$ belong to the same atom of $\bigvee_{-n_{-}}^{n_{+}} T^{k} P$, where $n_{ \pm}=\left[n /\left(\mu\left(F_{ \pm}\right)+2 \varepsilon\right)\right]$. Suppose $d(x, y) \leqq d(x, S)$. Then $x$ and $y$ belong to the same atom of $P$, and $d(T x, T y) \leqq F_{+}(x)$ $\cdot d(x, y)$. If, moreover, $d(x, y) \leqq d(T x, S) / F_{+}(x)$, we deduce $d(T x, T y) \leqq d(T x, S): x$ and $y$ belong to the same atom of $T^{-1} P$, and $d\left(T^{2} x \cdot T^{2} y\right) \leqq F_{+}(T x) \cdot F_{+}(x)$ $\cdot d(x, y)$. Similar arguments are valid for $T^{-1}$ and $F_{-}$. Thus, we see that a sufficient 
set of conditions to insure that $x$ and $y$ lie in the same atom of $\bigvee_{-n_{-}}^{n_{+}} T^{k} P$ is that

$$
\begin{cases}d(x, y) \leqq d\left(T^{k} x, S\right) / \prod_{j=0}^{k-1} F_{+}\left(T^{j} x\right), & 0 \leqq k \leqq n_{-}, \\ d(x, y) \leqq d\left(T^{-k} x, S\right) / \prod_{j=0}^{k-1} F_{-}\left(T^{-j} x\right), & 0 \leqq k \leqq n_{+}\end{cases}
$$

(where the product is taken equal to 1 if $k=0$ ).

We have the two following estimates:

1) Let $B\left(\alpha^{k}\right)$ be the neighborhood of $S$ of diameter $\alpha^{k}$. We recall that $\mu\left(B\left(\alpha^{k}\right)\right)$ $<K \cdot \alpha^{\tau k}$, where $K, \tau$ are two positive constants. Thus, if $\alpha<1$, the series $\sum_{k=0}^{\infty} \mu\left(B\left(\alpha^{k}\right)\right)$ converges. This allows us, through a measure theoretic result (see [L 3] e.g.) to derive the existence a.e. of a measurable function $C(x, \varepsilon), 0<C(x, \varepsilon) \leqq 1$ for $\mu$-a.e. $x \in \Omega$ such that for $k \in \mathbb{Z} d\left(T^{k} x, S\right) \geqq C(x, \varepsilon) e^{-|k| \varepsilon}$. In particular, we get

$$
\begin{cases}d\left(T^{k} x, S\right) \geqq C(x, \varepsilon) e^{-n_{-} \cdot \varepsilon}, & 0 \leqq k \leqq n_{-}, \\ d\left(T^{-k} x, S\right) \geqq C(x, \varepsilon) e^{-n_{+} \cdot \varepsilon}, & 0 \leqq k \leqq n_{+} .\end{cases}
$$

2) By the ergodic theorem, for $\mu$-a.e. $x \in \Omega, \exists \bar{N}(x)$ such that if $n_{-}>\bar{N}(x)$, $n_{+}>\bar{N}(x)$, we have

$$
\begin{cases}\prod_{j=0}^{k-1} F_{+}\left(T^{j} x\right) \leqq \exp \left(n_{-}\left(\mu\left(F_{+}\right)+\varepsilon\right)\right), & 0 \leqq k \leqq n_{-}, \\ \prod_{j=0}^{k-1} F_{-}\left(T^{-j} x\right) \leqq \exp \left(n_{+}\left(\mu\left(F_{-}\right)+\varepsilon\right)\right), & 0 \leqq k \leqq n_{+} .\end{cases}
$$

Assuming $\varepsilon$ is sufficiently small and setting $N(x)=\bar{N}(x) \cdot \mu\left(F_{+}+F_{-}\right)$, we get, for $n>N(x)$,

$$
\left\{\begin{aligned}
& d\left(T^{k} x, S\right) / \prod_{j=0}^{k-1} F_{+}\left(T^{j} x\right) \geqq C(x, \varepsilon) \cdot \exp \left[-n_{-}\left(\mu\left(F_{+}\right)+2 \varepsilon\right)\right]=C(x, \varepsilon) \cdot e^{-n} \\
& 0 \leqq k \leqq n_{-}, \\
& d\left(T^{-k} x, S\right) / \prod_{j=0}^{k-1} F_{-}\left(T^{-j} x\right) \geqq C(x, \varepsilon) \cdot \exp \left[-n_{+}\left(\mu\left(F_{-}\right)+2 \varepsilon\right)\right]=C(x, \varepsilon) \cdot e^{-n} \\
& 0 \leqq k \leqq n_{+} .
\end{aligned}\right.
$$

Then, $B\left(x, C(x, \varepsilon) \cdot e^{-n}\right) \subset\left(\bigvee_{-n_{-}}^{n_{+}} T^{k} P\right)(x)$ for $n>N(x)$.

Using Shannon-Mac Millan-Breimann's theorem (see [Bi]) we obtain for $\mu$-a.e. $x \in \Omega$,

$$
\liminf _{\alpha \rightarrow 0} \log \mu(B(x, \alpha)) / \log \alpha \geqq h \cdot\left(\frac{1}{\mu\left(F_{+}\right)+2 \varepsilon}+\frac{1}{\mu\left(F_{-}\right)+2 \varepsilon}\right) .
$$

As $\varepsilon$ is arbitrarily small, we have $\mu$-a.e.:

$$
\liminf _{\alpha \rightarrow 0} \log \mu(B(x, \alpha)) / \log \alpha \geqq h \cdot\left(\frac{1}{\mu\left(F_{+}\right)}+\frac{1}{\mu\left(F_{-}\right)}\right) .
$$


We can reproduce the same arguments replacing $T$ by $T^{n}, S_{0}^{ \pm}$by $\bigcup_{k=0}^{n-1} T^{ \pm k} S_{0}^{ \pm}, P$ by $\bigvee_{|k|<n} T^{k} P, F_{ \pm}$by $\left\|D T^{ \pm n}\right\|$.

As $\lim _{n \rightarrow \infty} \int\left\|D_{x} T^{ \pm n}\right\| d \mu(x)=\chi_{ \pm}$, we get

$$
\lim _{\alpha \rightarrow 0} \inf \log \mu(B(x, \alpha)) / \log \alpha \geqq h \cdot\left(\frac{1}{\chi_{+}}-\frac{1}{\chi_{-}}\right) .
$$

Lemma V.3. $\limsup _{\alpha \rightarrow 0} \log \mu(B(x, \alpha)) / \log \alpha \leqq h \cdot\left(\frac{1}{\chi_{+}}-\frac{1}{\chi_{-}}\right)$.

Proof. Let $P$ still denote the partition defined by $S$. We first prove that, $\varepsilon>0$ being given, there is a constant $K$ such that, for $\mu$-a.e. $x \in \Omega$, we can find $N(x)$ such that

$$
n>N(x) \Rightarrow\left(\bigvee_{-n_{-}}^{n_{+}} T^{k} P\right)(x) \subset B\left(x, K e^{-n}\right),
$$

where $n_{+}=n /\left[-\chi_{-}-\varepsilon\right], n_{-}=n /\left[\chi_{+}-\varepsilon\right]$.

Let $x \in \Omega$ and let $Q=\left(\bigvee_{-n_{-}}^{n_{+}} T^{k} P\right)(x)$.

We first exhibit "unstable and stable widths" $w_{ \pm}$of $Q$. For $y \in Q$, we draw the line $\Delta=\Delta(y)$ passing through $y$ in the unstable direction, and note $w_{+}(y)$ the length of $\Delta \cap Q$; we recall that $Q$ is a convex set. We set $w_{+}=\sup _{y \in Q} w_{+}(y)$ and define $w_{-}$ similarly. As the unstable and stable directions are transverse, and as the $S_{p}^{+}, p \in \mathbb{N}$, and the $S_{q}^{-}, q \in \mathbb{N}$, are also transverse, the minimum ball constaining $Q$ has a radius smaller than $C \cdot\left(w_{+}+w_{-}\right)$, where $C$ is a constant. We now estimate $w_{+}$; we fix $y \in Q$ such that $w_{+}(y)>w_{+} / 2 . T^{n-}$ is linear on $\Delta(y) \cap Q$. Thus, the usual argument shows that $10>l\left(T^{n_{-}}(\Delta(y) \cap Q)\right)=w_{+}(y) \cdot \prod_{i=0}^{n_{-}-1} J^{+}\left(T^{i} y\right)$, where $J^{+}$is the jacobian in the unstable direction. By the ergodic theorem, $\prod_{i=0}^{n_{-}-1} J^{+}\left(T^{i} y\right)=e^{n_{-}\left(\chi_{+}-\varepsilon\left(n_{-}\right)\right)}$, where $\varepsilon(\cdot)$ is a function depending on $y$ such that $\lim _{m \rightarrow \infty} \varepsilon(m)=0$. As $y$ is here fixed, for $n$ large enough, $n_{-}$is large enough and $\varepsilon\left(n_{-}\right)<\varepsilon$. We get $10>w_{+}(y) \cdot e^{n}$; similarly, $w_{-}(y)<10 \cdot e^{-n}$, and we get $\left(\bigvee_{-n_{-}}^{n_{+}} T^{k} P\right)(x) \subset B\left(x, K e^{-n}\right)$ with $K=20 \cdot C$. Letting $n$ go to infinity, we get

$$
\limsup _{\alpha \rightarrow 0} \frac{\log \mu(B(x, \alpha))}{\log \alpha} \leqq h\left(\frac{1}{\chi_{+}-\varepsilon}+\frac{1}{-\chi_{-}-\varepsilon}\right) .
$$

Lemma V.3 now follows from the fact that $\varepsilon$ is arbitrarily small, Lemmata V.2 and V.3 end the proof of Proposition V.1.

We add the following result, which is the mere consequence of $[\mathrm{L}, \mathrm{S}]$.

Proposition V.4. With the above notations, $h=\chi_{+}$.

Proof. One can check that hypothesis of $[\mathrm{K}, \mathrm{S}]$ are fulfilled, so that the result of $[\mathrm{L}, \mathrm{S}]$ applies. 


\section{Appendix. Proof of Proposition II.4}

Lemma A.1. There is a positive constant $L_{1}^{\prime}$ such that for any pair $W_{1}, W_{2}$ of local unstable manifolds in $\Omega$, there is an $\varepsilon_{0}, 0<\varepsilon_{0}<1$ such that if $0<\varepsilon<\varepsilon_{0}$, one can find an open subset $\mathscr{A}_{\varepsilon}$ of $\Omega$ with $l\left(\mathscr{A}_{\varepsilon} \cap W_{1}\right)<\frac{2 \varepsilon}{1-4 \sqrt{b} / \lambda}$, and if $\left.x, x^{\prime} \in \mathscr{D}\left(P_{W^{1} W^{2}}\right)\right)\left(\mathscr{A}_{\varepsilon}\right.$, $d(x, x)<\varepsilon^{2}$, then

$$
\left(1-L_{1}^{\prime} D^{1 / 3}\right) d\left(x, x^{\prime}\right) \leqq d\left(P_{W^{1} W^{2}}(x), P_{W^{1} W^{2}}\left(x^{\prime}\right)\right) \leqq d\left(x, x^{\prime}\right)\left(1+L_{1}^{\prime} D^{1 / 3}\right),
$$

where $D=d\left(W_{1}, W_{2}\right)$.

Proof. Let $K$ be a real number such that $\frac{2}{\lambda}<K<\frac{1}{\sqrt{b}}$. We define $\mathscr{A}_{\varepsilon}$ by

$$
\mathscr{A}_{\varepsilon}=\bigcup_{j=0}^{\infty}\left(A_{2 \varepsilon / K^{j}}^{-j} \cap \Omega\right), \quad \text { where } \quad A_{\alpha}^{-j}=T^{-j} A_{\alpha}^{-} .
$$

Every $A_{2 \varepsilon / K^{j}}^{-j}$ crosses $W_{1}$ at most $2^{j}$ times, therefore,

$$
\begin{aligned}
l\left(\mathscr{A}_{\varepsilon} \cap W_{1}\right) & \leqq \sum_{j=0}^{\infty} l\left(A_{2 \varepsilon / K^{j}} \cap W_{1}\right) \\
& \leqq 2 \sum_{j=0}^{\infty} 2^{j} \frac{1}{(K \lambda)^{j}} 2 \varepsilon=\frac{4 \varepsilon}{1-2 / K \lambda},
\end{aligned}
$$

because the stable and unstable manifolds are transverse. We note $p=P_{W^{1} W^{2}}$. Assume $x, x^{\prime} \in \mathscr{D}(p)$, and $x \notin \mathscr{A}_{\varepsilon}$ and $d\left(x, x^{\prime}\right) \leqq \varepsilon^{2}$. Let $\delta=d\left(x, x^{\prime}\right)$. Let $N$ be the integer such that $2 \varepsilon\left(\frac{1}{K \lambda_{+}}\right)^{N+1} \leqq \delta<2 \varepsilon\left(\frac{1}{K \lambda_{+}}\right)^{N}$. Assume that for some $k$, $0 \leqq k \leqq N, S_{k}^{-}$crosses $W_{1}$ at some point $y$ between $x$ and $x^{\prime}$. From $d(x, y)<\delta$, we deduce $d\left(T^{k} x, T^{k} y\right) \leqq \delta \lambda^{k}$. Therefore,

$$
d\left(T^{k} x, S_{0}\right) \leqq \delta \lambda_{+}^{k} \leqq \delta \lambda_{+}^{N} \leqq 2 \varepsilon K^{-N} \leqq 2 \varepsilon K^{-k},
$$

which contradicts $x \notin \mathscr{A}_{\varepsilon}$. Assume now that $S_{k}^{-}$crosses $W_{2}$ for some $k, 0 \leqq k \leqq N$, at some point which is on the segment $\left(p(x), p\left(x^{\prime}\right)\right)$. Since $S_{k}^{-}$cannot cross a local stable manifold, $S_{k}^{-}$must have a corner inside the parallelogram $Q=\left(x, x^{\prime}, p(x), p\left(x^{\prime}\right)\right)$. This implies that there is a point $z$ belonging to this parallelogram such that $z \in S_{k^{\prime}}^{-}$, for some $k^{\prime}, 0 \leqq k^{\prime}<k$. By induction we obtain that $Q$ must intersect $S_{0}^{-}$, a contradiction since $S_{0}^{-}$is a straight line. Let $j$ be an integer such that $0 \leqq j \leqq N$. The above argument implies that $T^{N-j} Q$ is a parallelogram. Moreover, we have

$$
d\left(T^{N-j} x, T^{N-j} x^{\prime}\right) \geqq \delta \lambda^{N-j}, d\left(T^{N-j} x, T^{N-j}(p(x))\right)<2\left(\frac{b}{\lambda}\right)^{N-j} D,
$$

and

$$
d\left(T^{N-j} x^{\prime}, T^{N-j}\left(p\left(x^{\prime}\right)\right)\right) \leqq 2\left(\frac{b}{\lambda}\right)^{N-j} D
$$


since the angle between a local stable and a local unstable manifold is greater than $\frac{\pi}{5}$. We have, therefore,

$$
\left|\frac{d\left(T^{N-j}(p(x)), T^{N-j}\left(p\left(x^{\prime}\right)\right)\right)}{d\left(T^{N-j}(x), T^{N-j}\left(x^{\prime}\right)\right)}-1\right|<10\left(\frac{b}{\lambda^{2}}\right)^{N-j} \frac{D}{\delta}
$$

which implies

$$
\left|\frac{d\left(T^{N-j}(p(x)), T^{N-j}\left(p\left(x^{\prime}\right)\right)\right)}{d\left(T^{N-j}(x), T^{N-j}\left(x^{\prime}\right)\right)}-1\right|<\frac{1}{10} \sqrt{D} \quad \text { by our choice of } N,
$$

provided $\varepsilon_{0}$ is chosen small enough (independently of $N$ and $\delta$ ), and $j \leqq \frac{1}{2} \frac{|\log D|}{\log \left(\lambda^{2} / b\right)}$. We also have

$$
\frac{d\left(T^{N-j}(p(x)), T^{N-j}\left(p\left(x^{\prime}\right)\right)\right)}{d\left(T^{N-j}(x), T^{N-j}\left(x^{\prime}\right)\right)} / \frac{d\left(p(x), p\left(x^{\prime}\right)\right)}{d\left(x, x^{\prime}\right)}=\prod_{l=1}^{N-j} \Delta_{l}
$$

where

$$
\Delta_{l}=\frac{d\left(T^{l}(p(x)), T^{l}\left(p\left(x^{\prime}\right)\right)\right)}{d\left(T^{l}(x), T^{l}\left(x^{\prime}\right)\right)} / \frac{d\left(T^{l-1}(p(x)), T^{l-1}\left(p\left(x^{\prime}\right)\right)\right)}{d\left(T^{l-1}(x), T^{l-1}\left(x^{\prime}\right)\right)}
$$

satisfies

$$
1-2\left(\frac{b}{\lambda}\right)^{N-l} \leqq \Delta_{l} \leqq 1+2\left(\frac{b}{\lambda}\right)^{N-l}
$$

according to Lemma I.1. Therefore, if $j=\left[\frac{1}{2} \frac{|\log D|}{\log \left(\lambda^{2} / b\right)}\right]-\log 8$, we have

$$
\left|\prod_{l=1}^{N-j} \Delta_{l}-1\right| \leqq \frac{\sqrt{D}}{2}, \quad \text { if } D \text { is smaller than } \frac{b}{64 \lambda^{2}}
$$

Combining the two estimates, we obtain the result for $D<\frac{b}{64 \lambda^{2}}$ with $L_{1}^{\prime}=1$. For $D>\frac{b}{64 \lambda^{2}}$, we apply $T$ one time [this is enough since $\left.D \leqq \mathcal{O}(1) b\right]$, and we can apply the estimate unless $W^{1}$ and $W^{2}$ cross $S_{0}$, in which case the estimation is performed with respect to $S_{0}$. Q.E.D.

We still note $p=P_{W^{1} W^{2}}$.

Proof of Proposition II.4. It is enough to prove the proposition with $A=\bar{A} \cap \mathscr{D}(p)$, $\bar{A}$ a closed interval in $W^{1}$ and $l(A)>0$. Let $\varepsilon_{0}$ be as in Lemma A.1, and choose $\varepsilon$ such that $0<\varepsilon<\varepsilon_{0}$ and $\varepsilon<l(A) D^{1 / 3}$. We observe that $W_{1} \backslash \mathscr{D}(p)$ is an open subset. Therefore, there is a sequence $\left(U_{k}\right)_{k \in \mathbb{N}}$ of disjoint open intervals such that

$$
W_{1} \backslash \mathscr{D}(p)=\bigcup_{k=0}^{\infty} U_{k}
$$


In the following, for $a, b \in \mathscr{D}(p)$ we shall denote by $\tilde{p}(] a, b[)$ the segment ]$p(a), p(b)[$ (although $p$ is not everywhere defined on $] a, b$ [, this definition makes sense because $p$ is order preserving).

Let $V_{k}=\tilde{p}\left(U_{k}\right)$. From $l\left(W_{1}\right)<\infty$ and $l\left(W_{2}\right)<\infty$, we deduce that there is an integer $N_{1}$ such that

$$
\sum_{k=N_{1}}^{\infty} l\left(U_{k}\right)<\varepsilon, \quad \sum_{k=N_{1}}^{\infty} l\left(V_{k}\right)<\varepsilon, \quad l\left(V_{k}\right)<\varepsilon^{2} \quad \text { if } \quad k \geqq N_{1} .
$$

Let $A^{\prime}=\bar{A} \backslash \bigcup_{k=0}^{N_{1}-1} U_{k}$. We have $l(A) \leqq l\left(A^{\prime}\right) \leqq l(A)+\varepsilon$ and $l(p(A)) \leqq l\left(\tilde{p}\left(A^{\prime}\right)\right)$. The set $\mathscr{A}^{\prime}=W_{2} \cap \mathscr{A}_{\varepsilon} \cup\left(\bigcup_{k=N_{1}}^{\infty} V_{k}\right)$ is an open subset of $W_{2}$ such that

$$
l\left(\mathscr{A}^{\prime}\right) \leqq\left(1+\frac{2}{1-4 \sqrt{b} / \lambda}\right) \varepsilon \leqq 4 \varepsilon \quad \text { if } b \text { is small enough } .
$$

We can find a sequence $\left(V_{k}^{\prime}\right)_{k \in \mathbb{N}}$ of disjoint open intervals of $W_{2}$ such that

$$
\mathscr{A}^{\prime}=\bigcup_{k=0}^{\infty} V_{k}^{\prime} \text {. }
$$

Let $N_{2}$ be an integer such that

$$
l\left(V_{k}^{\prime}\right)<\varepsilon^{2} \quad \text { if } \quad k \geqq N_{2} \quad \text { and } \quad \sum_{k=N_{2}}^{\infty} l\left(V_{k}^{\prime}\right)<\varepsilon .
$$

Let

$$
A^{\prime \prime}=\widetilde{P}^{-1}\left(\widetilde{P}\left(A^{\prime}\right) \mid \bigcup_{k=0}^{N_{2}-1} V_{k}^{\prime}\right)
$$

we have $l\left(A^{\prime \prime}\right) \leqq l\left(A^{\prime}\right)$ and $l\left(\tilde{p}\left(A^{\prime \prime}\right)\right) \leqq l\left(\tilde{p}\left(A^{\prime}\right)\right) \leqq l\left(\tilde{p}\left(A^{\prime \prime}\right)\right)+4 \varepsilon . \tilde{p}\left(A^{\prime \prime}\right)$ is a finite union of closed intervals of $W_{2}$ whose endpoints are in $\mathscr{D}\left(p^{-1}\right) \backslash \mathscr{A}_{\varepsilon}$. Let $I=[u, v]$ be such an interval. We claim that there is a finite sequence $\left(u_{s}\right)_{s=0, \ldots, q}$ such that

i) $u_{0}=u, u_{q}=v$,

ii) $u_{j} \in \mathscr{D}\left(p^{-1} \backslash \mathscr{A}_{\varepsilon}\right)$,

iii) $d\left(u_{j}, u_{j+1}\right) \leqq \varepsilon^{2}$.

This is obvious from the above construction. From Lemma A.2.1, we have

$$
l\left(\tilde{p}^{-1}\left[u_{j}, u_{j+1}\right]\right) \leqq\left(1+L_{1}^{\prime} D^{1 / 3}\right) l\left[u_{j}, u_{j+1}\right] \forall j, \quad 0 \leqq j \leqq q-1,
$$

which implies

$$
l\left(\tilde{p}^{-1}(I)\right) \leqq\left(1+L_{1}^{\prime} D^{1 / 3}\right) l(I) \text { and } \quad l\left(A^{\prime \prime}\right) \leqq\left(1+L_{1}^{\prime} D^{1 / 3}\right) l\left(\tilde{p}\left(A^{\prime \prime}\right)\right) .
$$

Therefore,

$$
\begin{aligned}
l(p(A)) & \leqq l\left(\tilde{p}\left(A^{\prime}\right)\right) \leqq l\left(\tilde{p}\left(A^{\prime \prime}\right)\right)+4 \varepsilon \leqq\left(1+L_{1}^{\prime} D^{1 / 3}\right) l\left(A^{\prime \prime}\right)+4 \varepsilon \\
& \leqq\left(1+L_{1}^{\prime} D^{1 / 3}\right) l\left(A^{\prime}\right)+4 \varepsilon \leqq\left(1+L_{1}^{\prime} D^{1 / 3}\right) l(A)+\left(5+L_{1}^{\prime} D^{1 / 3}\right) \varepsilon \\
& \leqq\left(1+L_{1} D^{1 / 3}\right) l(A), \quad \text { where } \quad L_{1}=2 L_{1}^{\prime}+5 .
\end{aligned}
$$


The inequality in the other direction is obtained by interchanging $W_{1}$ and $W_{2}$. Q.E.D.

Acknowledgement. We would like to thank J. Lascoux and F. Ledrappier for many helpful discussions.

\section{References}

[B] Bourbaki, M.: Eléments de Mathématiques, Integration chapter IX. Paris: Hermann 1969

[Bi] Billingsley, P.: Ergodic theory and information. New York: Wiley 1965

[He] Henon, M.: A two-dimensional mapping with a strange attractor. Commun. Math. Phys. 50, 69-77 (1976)

[K, S] Katok, A., Strelcyn, J.M.: Invariant manifolds for smooth maps with singularities, Part I: Existence. Preprint (1980)

[L1] Ledrappier, F.: Propriétés ergodiques des mesures de Sinaï. Preprint (1982)

[L2] Ledrappier, F.: Sur la condition de Bernoulli faible et ses applications. Théorie ergodique Rennes 73/74. In: Lecture Notes in Mathematics, Vol. 532. Berlin, Heidelberg, New York: Springer 1975

[L3] Ledrappier, F.: Quelques propriétés des exposants caractéristiques. Ecole d'été de probabilités de St. Flour 1982. In: Lecture Notes in Mathematics. Berlin, Heidelberg, New York: Springer 1983 (to appear)

[L,S] Ledrappier, F., Strelcyn, J.M.: A proof of the estimation from below in Pesin entropy formula. Preprint. Paris-Nord (1982)

[Lo] Lozi, R.: Un attracteur étrange du type attracteur de Henon. J. Phys. (Paris) 39 (Coll. C5), 9-10 (1978)

[M] Misiurewicz, M.: Strange attractors for the Lozi mappings. In: Nonlinear dynamics, R. G. Helleman (ed.). New York: The New York Academy of Sciences 1980

[P] Parry, W.: Entropy and generators in ergodic theory. New York: Benjamin 1969

[Ro] Rohlin, V.A.: On the fundamental ideas of measure theory. Math. Sbornik 25 (67), 107-150 (1949)

[Ry] Rychlik, M.: Théorie ergodique, mesures invariantes et principe variationnel pour les applications de Lozi. C. R. Acad. Sci. Paris (1983) (to appear)

[Y1] Young, Lai-Sang: Bowen-Ruelle measures for certain piecewise hyperbolic maps. Preprint, University of Wisconsin (1982)

[Y2] Young, Lai-Sang: Dimension, entropy, and Lyapunov exponents. Ergod. Theor. Dynam. Syst. 2, 109-124 (1982)

Communicated by J. Lascoux

Received June 13, 1983; in revised form November 1, 1983 
